\title{
Aplicação do Operador OWA nos Problemas da Otimização de Ângulo de Feixe e da Intensidade em IMRT
}

\author{
Sidemar F. Cezario ${ }^{1}$, Elizabeth F. G. Goldbarg ${ }^{1}$, Sílvia M. D. M. Maia ${ }^{1}$ \\ ${ }^{1}$ Departamento de Informática e Matemática Aplicada \\ Universidade Federal do Rio Grande do Norte (UFRRN) - Natal - RN - Brasil \\ sidemar@ppgsc.ufrn.br, \{beth,silvia\}@dimap.ufrn.br
}

\begin{abstract}
Radiation therapy is an extremely important method for cancer treatment. The main challenge is to deliver, at least, the prescribed dose to the tumor, while avoiding to expose healthy organs to radiation beyond defined limits. The intensity modulated teletherapy (IMRT) is an advanced mode of high-precision radiotherapy. The main difficulty of IMRT treatments consists in automating the selection of beam angles for an adequate dose distribution. This research presents an algorithm that seeks the ideal balance between a set of angles and a dose distribution that respects medical prescriptions inherent to the treatment. The proposed algorithm uses two new mathematical models and the Ordered Weighted Average (OWA) operator as a criterion of preference to choose the best solution.
\end{abstract}

Resumo. A radioterapia é um método extremamente importante no tratamento do câncer. O principal desafio é entregar pelo menos a dose prescrita para o tumor, enquanto evita expor os órgãos saudáveis à radiação além do permitido. Um dos principais modos de tratamento nesta área é a teleterapia de intensidade modulada (IMRT). A principal dificuldade da IMRT consiste em automatizar a seleção de ângulos de feixes para uma melhor distribuição de dose. Nesta pesquisa é apresentado um algoritmo que busca o balanceamento ideal, entre um conjunto de ângulos e uma distribuição de dose que respeite as prescrições médicas inerentes ao tratamento. O algoritmo proposto utiliza dois novos modelos matemáticos e o operador Ordered Weighted Average (OWA) como critério de preferência na escolha da melhor solução.

\section{Introdução}

O Instituto Nacional do Câncer, por meio do seu relatório intitulado Incidência de Câncer no Brasil [INCA 2018] mostra um crescimento anual nos casos de câncer. Estima-se que em 2012, aconteceram 14,1 milhões de novos casos de câncer e 8,2 milhões de óbitos, a maioria desses casos atingiram pessoas do sexo masculino que vivem em países desenvolvidos. De acordo com [Breedveld et al. 2018], o câncer é diagnosticado em cerca de 15 milhões de pacientes a cada ano, e em $50 \%$ dos casos a radioterapia é usada, às vezes em combinação com quimioterapia ou cirurgia. Em aproximadamente $40 \%$ dos casos curados, envolve a utilização de radioterapia.

Os problemas de otimização no planejamento de teleterapia de intensidade modulada (IMRT), consiste em basicamente três. O primeiro é o problema de otimização de ângulo de feixe (OAF), que se refere a escolha dos posicionamentos dos campos que a 
radiação será emitida. O próximo problema é a otimização do mapa de fluências (OMF), que determina quanto de dose deverá ser emitida. Por fim, o problema de realização que busca como configurar o colimador multilâminas para entregar a dose planejada. $\mathrm{O}$ foco deste trabalho está nos dois primeiros problemas de otimização.

\section{Definição do Problema}

Segundo [Cabrera et al. 2016], o problema da OAF pode ser definido da seguinte forma.

$$
\min _{\mathcal{A} \in \mathcal{P}^{N}(K)} h(\mathcal{A})
$$

- Sendo $K$ o conjunto de ângulos, geralmente $K=\{k \pi / 180: k \in\{1, \ldots, 359\}\}$.

- $\mathcal{P}^{N}(K)=\{x \subseteq K:|x|=N\}$, sendo $\mathcal{P}^{N}(K)$ subconjuntos de $K$ e $N$ a cardinalidade do conjunto $x$.

O problema da OAF tem como objetivo encontrar uma configuração de ângulo de feixe (CAF), que miniminiza uma função $h: \mathcal{P}^{N} \rightarrow \mathbb{R}^{q}$, sendo $q$ o número de objetivos. É importante notar que esse é um problema NP-difícil [Sultan and Saher 2006].

A respeito do OMF, a equação (2) apresenta uma formulação proposta em [Cabrera et al. 2016]:

$$
f(\mathcal{A})=\min _{x \in X(\mathcal{A})} z(x)
$$

- $z(x) \in \mathbb{R}^{Q}$ é um vetor com $Q$ funções objetivo $z_{q}$, com $q \in\{1, \ldots, Q\}$, onde $Q$ é o número de órgãos considerados no tratamento.

- $x \in \mathbb{R}_{\geq 0}^{n}$ é o vetor de número reais não negativos que corresponde ao mapa de fluência.

- dada uma CAF, $X(\mathcal{A})$ é o conjunto das soluções viáveis do problema OMF.

A dose depositada $d$ em cada órgão, incluindo o tumor é calculado através da expressão (3). A é a matriz de deposição que contém a taxa de radiação da região e $x$ é o mapa de fluência, ou seja, o tempo de exposição da radiação na região.

$$
d=A x
$$

\section{Solução Proposta e Contribuições}

O operador OWA [Fernández et al. 2014] vem destacando-se em problemas de otimização multiobjetivo que tem como características: grande quantidade de objetivos; dificuldade em atribuir pesos aos objetivos e encontrar uma solução significativa. Um exemplo de problema com essas características, é a árvore geradora multiobjetivo, onde os trabalho de [Galand and Spanjaard 2012] e [Fernández et al. 2017], exploraram o OWA. O problema de OAF possui exatamente essas mesmas características e até que se tenha conhecimento o OWA nunca foi testado em problemas referentes a radioterapia. Diante disso, essa pesquisa combina o OWA com modelos do problema de OAF, criando uma solução que contribui para diminuir o tempo necessário do planejamento no tratamento de câncer. 
O primeiro modelo proposto nessa pesquisa resolve o problema de OMF, e tem como principal característica minimizar as folgas de dose de todos os órgãos e PTV. Sua estrutura é detalhada como segue.

Parâmetros

- $A^{r}$ é a matriz que contém as taxa de radiação que passa por cada sub-feixe, aplicada aos voxels da região $r$, ou seja, cada elemento $a_{i j} \in A^{r}$ denota a taxa de radiação do sub-feixe $i$ no voxel $j$ do órgão $r$.

- $r \in R=\left\{T, O_{1}, \ldots, O_{Q}\right\}$ indica uma região do conjunto de regiões $R$, ou seja, cada órgão do problema. $T$ é a região do tumor e o $O_{k}$, indica o $k$-ésimo órgão.

- $m^{t}$ é o numero de voxels do PTV.

- $m^{r}$ é o numero de voxels do órgão $r$.

- $d_{p m i n}^{t}$ é a dose mínima prescrita para cada voxel do PTV.

- $d_{\text {pmax }}^{t}$ é a dose máxima para cada voxel do PTV.

- $d_{p \max }^{r}$ é a dose máxima para cada voxel do órgão $r$.

- $n$ é o número de sub-feixes.

Variáveis

- $f$ é a folga ou deficit de dose para cada voxel do PTV.

- $g^{r}$ é a folga ou excesso de dose para cada voxel da região $r$.

- $x$ é o mapa de intensidade.

- $d^{t}$ é um vetor de dose, onde seus elementos $d^{t}$ representam a dose total depositada nos voxels da região do PTV pelo mapa de intensidades $x$.

- $d^{r}$ é um vetor de dose, onde seus elementos $d^{r}$ representam a dose total depositada nos voxels da região $r$ pelo mapa de intensidades $x$.

$$
\begin{aligned}
& \min \sum_{i=1}^{m^{t}} f_{i} \\
& \min \sum_{i=1}^{m^{r}} g_{i}^{r} \quad \forall r \in R \backslash\{T\}
\end{aligned}
$$

Sujeito à:

$$
\begin{array}{rc}
d^{r}=A^{r} x & \forall r \in R \\
d_{i}^{t} \leq d_{\text {max }}^{t} & i=1, \ldots, m^{t} \\
d_{i}^{t} \geq d_{\text {mmin }}^{t}-f_{i} & i=1, \ldots, m^{t} \\
d_{i}^{r} \leq d_{p \max }^{r}+g_{i}^{r} & \forall r \in R \backslash\{T\} \quad i=1, \ldots, m^{r} \\
f_{i} \geq 0 & i=1, \ldots, m^{t} \\
g_{i}^{r} \geq 0 & i=1, \ldots, m^{r} \\
x_{i} \geq 0 & i=1, \ldots, n
\end{array}
$$

As funções objetivo do modelo OMF1, representado pelas fórmulas (4) e (5), visa minimizar as folgas $f$ e $g^{r}$, respectivamente, deficit de dose nos voxels do tumor e excesso de dose nos voxels das demais regiões. Assim o número de objetivos desse modelo é justamente a quantidade de órgãos do paciente que devem ser considerados. A expressão 
(6) contribui de forma significativa para o desempenho em tempo computacional do modelo, permitindo que o processo de otimização seja acelerado pelo solver. A restrição (7) garante uma dose máxima para cada voxel da região do câncer (PTV). A restrição (8) admite que exista uma folga entre a dose mínima prescrita para cada voxel do PTV e a dose obtida, de forma semelhante, a restrição (9) permite que exista uma folga entre a dose máxima prescrita e a dose encontrada para cada voxel dos órgãos sadios. O ideal é que essa folga seja zero, assim a solução obtida é ideal para o tratamento do paciente. Por último, as restrições (10)-(11) garantem a não negatividade das folgas e a (12) garante a não negatividade do mapa de intensidade.

Com o intuito de flexibilizar a dose no PTV, dando prioridade aos demais órgãos, foi criado um segundo modelo OMF2. Esse modelo segue a mesma estrutura de parâmetros e variáveis do anterior, porém o OMF2 garante que não ocorrerá nenhum excesso de radiação aos órgãos saudáveis. Para isso, foram removidas as expressões (5), (9) e (11) e adicionada a (13).

$$
d_{i}^{r} \leq d_{p \max }^{r} \quad \forall r \in R \backslash\{T\} \quad i=1, \ldots, m^{r}
$$

A restrição (13) garante que não existirá excesso de dose em nenhum voxel dos órgãos saudáveis, respeitando assim a prescrição.

Este trabalho propõe um algoritmo que visa encontrar uma solução viável com o menor número de ângulos possível, isso torna o tratamento mais simples e mais rápido. Para isso, o algoritmo utiliza uma estratégia gulosa. Primeiramente, busca-se descobrir qual ângulo obtém o menor valor objetivo, ou seja, a configuração de ângulo de feixe formada por apenas um ângulo que possui o menor valor objetivo usando o modelo OMF1, expressões (4)-(12), em conjunto com o modelo OWA. Em seguida, busca-se encontrar um segundo ângulo que unido com o primeiro gera o menor valor objetivo, e assim por diante. Caso a adição de um novo ângulo não minimize o valor objetivo ou o limite máximo de ângulos permitidos seja alcançado, o algoritmo é finalizado. O modelo OMF2 somente será utilizado em instâncias que a solução encontrada está excedendo a dose prescrita em órgãos sadios.

\section{Resultados}

As instâncias utilizadas nesse trabalho foram as publicadas no trabalho de [Breedveld and Heijmen 2017]. A base contém um conjunto de informações de 120 pacientes, divididos em três diferentes tipos de tumores: próstata, fígado e cabeça-pescoço. As instâncias podem ser baixadas em https://www6.erasmusmc.nl/radiotherapie/research2/medical-physics/projects/trots/.

A Figura 1 apresenta apenas alguns histogramas obtidos através do algoritmo proposto, sendo os HDVs de (a)-(d) são referentes as instâncias de câncer no fígado, enquanto que os HDVs de (e)-(h) são de câncer na próstata. Os demais HDVs são bastante semelhantes aos apresentados na Figura 1.

Analisando todas as 20 soluções encontradas para as instâncias, notou-se que o algoritmo se mostrou capaz de encontrar boas soluções em 15 instâncias. além disso, o algoritmo utilizava poucos ângulos (muitas soluções usavam menos de 3, dos 15 ou 25 disponíveis nas instâncias), acelerando assim o tratamento do câncer e proporcionado 


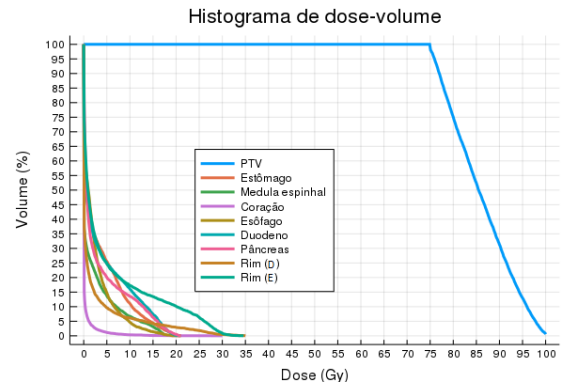

(a) HDV 01

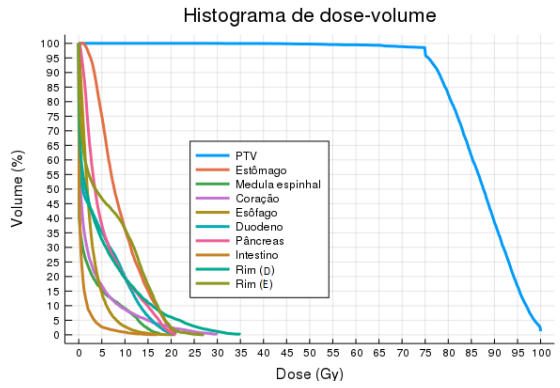

(c) HDV 03

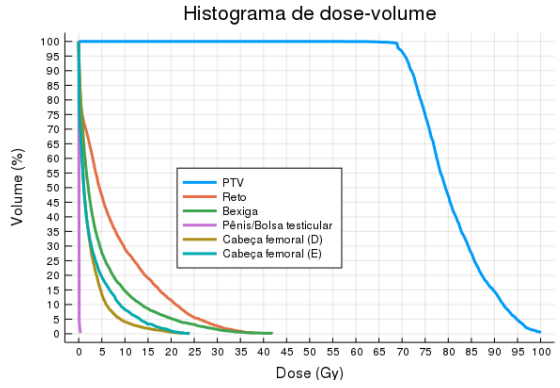

(e) HDV 05

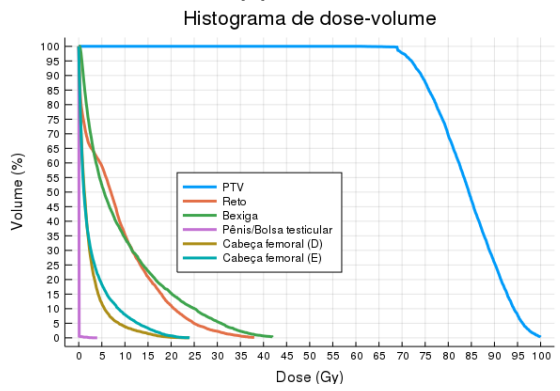

(g) HDV 07

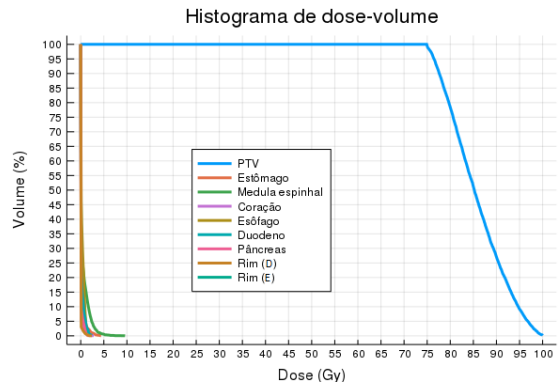

(b) HDV 02

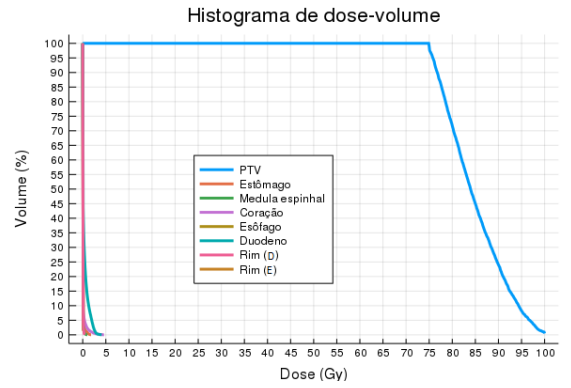

(d) HDV 04

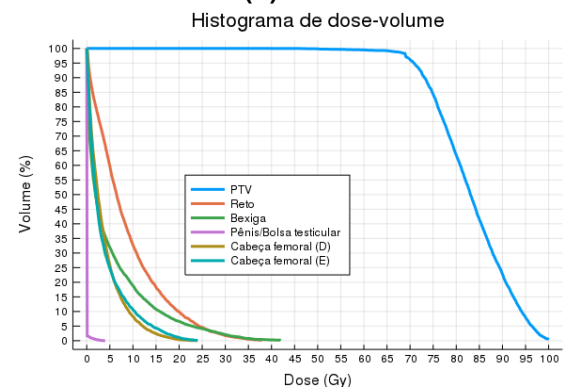

(f) HDV 06

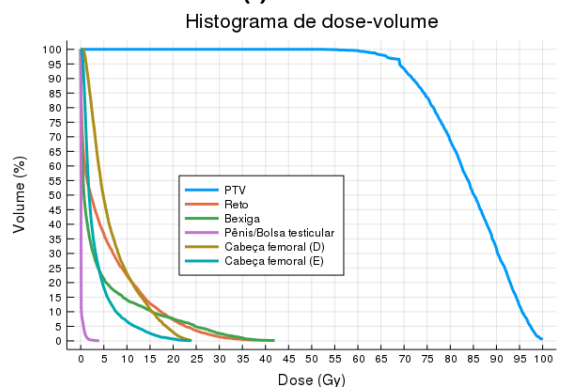

(h) HDV 08

Figura 1. Diagramas de dose-volume de algumas soluções encontradas pelo algoritmo proposto na pesquisa.

ganho de tempo, sendo o tempo um fator muito importante, pois com isso mais pessoas podem ser atendidas. Outra característica notada no algoritmo é a baixa dosagem que atingia os órgãos sadios, evitando criar complicações futuras.

\section{Conclusão}

Com base nos resultados apresentados, percebe-se que a utilização do OWA mostrou-se promissora para o Problema da Otimização de Ângulo de Feixe. O algoritmo foi testado em vinte instâncias, obtendo resultados viáveis para quinze desses casos de teste. 
Além disso, este trabalho torna-se inovador por introduzir um algoritmo que usa Ordered Weighted Average em modelos de radioterapia envolvendo a OAF.

Apesar dos bons resultados, é necessário aprimorar o algoritmo, visto que em cinco instâncias, o mesmo não conseguiu encontrar soluções boas para esses casos de teste. Outra atividade importante a ser realizada, é a execução de mais experimentos em instâncias referentes ao câncer em outras regiões além do fígado e próstata.

\section{Referências}

Breedveld, S., Craft, D., Van Haveren, R., and Heijmen, B. (2018). Multi-criteria optimisation and decision-making in radiotherapy. European Journal of Operational Research.

Breedveld, S. and Heijmen, B. (2017). Data for trots-the radiotherapy optimisation test set. Data in brief, 12:143-149.

Cabrera, G., Ehrgott, M., Mason, A. J., Raith, A., et al. (2016). A matheuristic approach to solve the multiobjective beam angle optimization problem in intensity-modulated radiation therapy. International Transactions in Operational Research.

Fernández, E., Pozo, M. A., and Puerto, J. (2014). Ordered weighted average combinatorial optimization: Formulations and their properties. Discrete Applied Mathematics, 169:97-118.

Fernández, E., Pozo, M. A., Puerto, J., and Scozzari, A. (2017). Ordered weighted average optimization in multiobjective spanning tree problem. European Journal of Operational Research, 260(3):886-903.

Galand, L. and Spanjaard, O. (2012). Exact algorithms for owa-optimization in multiobjective spanning tree problems. Computers \& Operations Research, 39(7):1540-1554.

INCA (2018). Incidência de Câncer no Brasil. http:www.inca.gov.br/ estimativa/2018/estimativa-2018 .pdf. [Online; acessado em 15-Nov2018].

Sultan, A. and Saher, A. (2006). Optimization of beam orientation in intensity modulated radiation therapy planning. 